\title{
Calidad de las tesis de maestría en temas de salud pública. Estudio en una universidad pública de Lima, Perú
}

\author{
Hernán A. Sanabria-Rojas, Carolina B. Tarqui-Mamani, Eduardo E. Zárate-Cárdenas
}

Objetivo. Evaluar la calidad de las tesis de maestría en el campo de la salud pública sustentadas por maestrandos de la unidad de posgrado de una facultad de medicina de Lima, Perú.

Materiales y métodos. Se revisaron 47 tesis que cumplían con los criterios de inclusión provenientes de maestrías cuyos temas de tesis se relacionaban con la salud pública. Las tesis se obtuvieron de la biblioteca de la facultad de medicina en estudio. Para la evaluación de la calidad de las tesis se estructuró la siguiente escala: excelente, aceptable y no aceptable; para ver la relevancia social también se elaboró una escala según el número de beneficiarios. La investigación se realizó en el segundo semestre del año 2008 y se realizó por dos evaluadores.

Resultados. Once (23\%) de las tesis fueron excelentes, cinco (11\%) fueron aceptables, y el resto, 31 (66\%), no aceptables. Del total de 11 tesis excelentes, cuatro correspondieron a la maestría en epidemiología, y cuatro, a salud reproductiva. De las 31 tesis no aceptables, el mayor porcentaje (23\%) correspondió a la maestría en gerencia de servicios de salud.

Conclusión. Poco más de un tercio de las tesis de maestría relativas a salud pública de la unidad de posgrado de la facultad de medicina en estudio son excelentes o aceptables en cuanto a su calidad metodológica, y sólo una minoría de dichas tesis de maestría son socialmente irrelevantes.

Palabras clave. Calidad. Maestría. Perú. Salud pública. Tesis. Universidad.

Quality of Public Health Masters' theses subjects. A study in a public university in Lima, Peru

Aim. To evaluate the quality of Public Health Masters' theses sustained by master candidates from a School of Medicine in Lima, Peru.

Materials and methods. We reviewed 47 theses which met the inclusion criteria. Theses came from masters programs and focused on public health issues. All the theses were obtained from the medical school library. Scale categories for quality evaluation ranged from excellent to acceptable, and unacceptable. Additionally, we used another scale to evaluate social relevance was based on the number of beneficiaries. The study was conducted in the second half of 2008.

Results. A total of 11 (23\%) were excellent, 5 (11\%) were acceptable, and 31 (66\%) were not acceptable. From the 11 excellent theses, four were form the Master of Epidemiology Program, and four were from the Master in Reproductive Health. From the 31 unacceptable theses, most of them (23\%) were from the Master of Healthcare Management Program.

Conclusion. Around a third of master theses with public health subjects from the Post-Graduate Unit in the School of Medicine are excellent or acceptable regarding the quality of their methods, and a minority were socially irrelevant.

Key words. Master. Peru. Public health. Quality. Thesis. University.

\section{Introducción}

El ser humano siempre ha tenido un concepto intuitivo de la calidad en razón de la búsqueda y el afán de perfeccionamiento como constantes de sus obras a través de la historia. La raíz etimológica de la palabra 'calidad' está en el término griego kalos, que significa lo bueno, lo apto, y también en la pala- bra latina qualitatem, que significa cualidad o propiedad [1].

La calidad educativa es entendida como el máximo grado educativo que se espera alcanzar en una área del conocimiento y tiene como fin lograr una alta especialización profesional, es decir, el dominio del conocimiento [1]. La calidad también alcanza la investigación científica que se realiza para la obten-
Departamento de Medicina Preventiva y Salud Pública. Facultad de Medicina. Universidad Nacional Mayor de San Marcos. Lima, Perú.

Correspondencia:

Prof. Hernán Arturo Sanabria Rojas. Facultad de Medicina de San Fernando. Universidad Nacional Mayor de San Marcos. Avda. Grau, 755. Lima 1, Perú.

E-mail:

hsanabriar@unmsm.edu.pe

(c) 2011 Educación Médica 
ción de grados universitarios, que se denomina tesis, las cuales son susceptibles de evaluarse.

La universidad se encarga de brindar una formación superior en todos sus niveles para que la sociedad avance y se desarrolle y, por lo tanto, es responsable de formar investigadores. La formación del magister o maestro culmina con la realización de la tesis y la obtención del grado de maestría según lo estipulado por la universidad. Sin embargo, la obtención del grado de maestría no garantiza que realmente se ha formado un investigador que impulsará el avance científico. En muchos casos podría ser cierto, pero en otros seguramente no lo sea. Los 'productos' más importantes de toda universidad son indudablemente sus profesionales formados en sus diferentes facultades y escuelas de pre y posgrado; en consecuencia, la evaluación de estos profesionales es mandatoria. La evaluación de la producción científica en las universidades es poco frecuente porque se ha orientado a evaluar mayormente el rendimiento de los alumnos; sin embargo, es un elemento clave que permite realizar el control de calidad sobre cómo se está realizando la investigación.

El proceso de globalización en los últimos años ha llevado a las universidades peruanas a ingresar a un mercado nacional e internacional muy competitivo; las investigaciones sobre la calidad de las tesis universitarias en el área de salud son muy escasas en Perú. Al respecto, en la Universidad Nacional de Trujillo, Narváez [2] dio a conocer una primera experiencia evaluando tesis; asimismo, Falcón et al [3] evaluaron las tesis de bachiller en la Facultad de Ciencias Médicas de la misma universidad, y finalmente, Veneros et al [4] hicieron lo propio con las tesis de la Facultad de Ciencias Biológicas. En otras universidades nacionales, Núñez-Sánchez y KalaConza [5] evaluaron la calidad de las tesis de bachiller en la Universidad Nacional San Antonio Abad del Cusco (UNSAAC). En la Universidad Nacional Mayor de San Marcos (UNMSM), Sanabria y Bullón [6] evaluaron la calidad de las tesis de bachiller en enfermería, mientras que Kredert [7] hizo lo propio en la Universidad Nacional del Centro (UNC), en Huancayo.

En la Unidad de Posgrado (UPG) de la Facultad de Medicina donde se hizo este estudio se forman maestros en diversas áreas de la salud pública, siendo la tesis su producto para que los maestrandos obtengan su grado de magister. Los candidatos cumplen con exigencias que impone la universidad antes de que defiendan sus tesis públicamente: 72 créditos del plan de estudios de la maestría aprobados, inscripción del proyecto de tesis revisado por un asesor, declaración de expedito administrativamen- te, revisión de la tesis por un jurado informante de tres evaluadores y, luego, revisión y sustentación de la tesis ante un jurado examinador de cinco evaluadores; finalmente la universidad expide una resolución rectoral que otorga el grado de magister.

Las áreas consideradas para el estudio fueron las maestrías en salud pública, epidemiología, gerencia en servicios de salud, salud reproductiva, salud ocupacional y ambiental, entre otras. El estudio se enmarca dentro de los conceptos de la gestión y calidad de la educación porque la calidad de la tesis de magister es un indicador objetivo que refleja el proceso de formación del estudiante de maestría para lograr dicho grado. Los resultados de la calidad de las tesis del área de salud pública servirán a las autoridades correspondientes para incorporar estrategias educativas que permitan mejorar la calidad de las futuras tesis.

El objetivo de la investigación fue evaluar la calidad de las tesis en áreas de la salud pública y determinar la relevancia social de las tesis correspondientes a las diferentes maestrías que ofrece la UPG de la Facultad de Medicina de una reconocida universidad pública de Lima, Perú; concomitantemente, se indagó si las investigaciones tenían una orientación de prevención y control, de gestión u otros relacionados con la salud.

\section{Materiales y métodos}

Se realizó un estudio observacional y descriptivo entre agosto de 2008 y enero de 2009. Se incluyeron 47 tesis registradas entre 1999 y el primer semestre del año 2008. Los criterios de inclusión fueron: tesis para optar al grado de maestro o magister con títulos de las diversas áreas de la salud pública y que fueron sustentadas en la UPG de la Facultad de Medicina en estudio, desde que se inició el programa de maestría en el año 1999. Se excluyeron de la evaluación las tesis sustentadas después del primer semestre de 2008 porque no se encontraban en la biblioteca de la facultad. La recolección de los datos incluyó, en primer lugar, la búsqueda en el registro de tesis que obran en la referida UPG; seguidamente, y en base al registro, se solicitó la versión electrónica de los resúmenes de todas las tesis de maestría ubicadas en la biblioteca central de la Facultad de Medicina; posteriormente se seleccionaron las tesis cuyos contenidos se referían a temas de salud pública.

Se estudiaron cuatro indicadores de calidad validados en un estudio previo por uno de los autores [6]: problema científico, muestra, análisis (comparabilidad) y conclusiones, como pilares de la inves- 
tigación científica. Para la evaluación de la calidad de las tesis se estructuró una escala ordinal con las siguientes denominaciones de valor:

- Excelente: si el autor de la tesis planteó o formuló un problema de investigación, tuvo una muestra representativa y adecuada, llegando a conclusiones tras hacer correctamente el análisis.

- Aceptable: si se planteó o formuló un problema de investigación, si se obtuvo una muestra representativa y adecuada, llegando a conclusiones sin hacer completamente el análisis, es decir, solamente opiniones y análisis estadísticos del propio autor de la tesis.

- No aceptable: cuando la tesis no se consideró excelente o aceptable tras la evaluación.

Se consideró un problema científico correcto si hubo una formulación explícita de un problema por investigar a manera de pregunta, o bien si estuvo planteado como objetivo. En cuanto a la muestra, ésta debía ser representativa y adecuada, es decir, proveniente de la población y explicitado el tamaño muestral. Un análisis correcto implicó la existencia del atributo 'comparabilidad', esto es, que el análisis se hubiera hecho según el plan de análisis señalado en la sección de materiales y métodos, o bien establecer comparaciones con los resultados de otros investigadores, y no solamente opiniones y análisis estadísticos del propio autor en la discusión. Para considerar correcta la conclusión, debía plantearse una generalización a la población objetivo a partir de una muestra apropiada debidamente analizada; en el caso de estudios descriptivos se consideró que había conclusión si la tesis presentaba un enunciado resumen y no propiamente una inferencia.

Para la evaluación de la relevancia social, ésta se hizo acorde a la población potencialmente beneficiaria en el estudio. Se utilizó la escala siguiente:

- Relevante: utilidad para más de 500 personas.

- Poco relevante: utilidad para 50 o más personas y menos de 500.

- Irrelevante: utilidad para menos de 50 personas.

La recolección de datos fue realizada por el equipo de investigación, a través de una ficha confeccionada ex profeso, en la biblioteca de la Facultad de Medicina en estudio. Seguidamente, se realizó una doble entrada de los datos en Microsoft Excel para detectar datos inconsistentes y proceder a la revisión de la ficha y realizar las correcciones pertinentes. La evaluación fue realizada por dos evaluadores y su concordancia entre evaluadores se realizó utilizando el estadístico kappa ( $\kappa=0,90 ; p=0,046)$. La información se procesó mediante el programa SPSS v. 15.0.

\section{Resultados}

El mayor número de tesis sustentadas se observó en los últimos tres años del estudio: 26 en el año 2006, 21 en el 2007 y 22 en el 2008. El 30\% de las tesis sustentadas en el área de salud pública correspondían al año 2006. Cabe precisar que sólo se evaluó una tesis sustentada en el año 2008 porque la recolección de datos fue hasta el mes de octubre y las tesis no suelen llegar a la biblioteca inmediatamente después de las sustentaciones de grado.

Del total de tesis sustentadas, el 30\% $(n=14)$ correspondían a salud reproductiva; el $20 \%(n=9)$, a epidemiología; el 15\% $(n=7)$, a gestión/gerencia de servicios; el 11\% $(n=5)$, a salud pública; el $11 \%$ $(n=5)$, a enfermería; el $6 \%(n=3)$, a salud ocupacional y ambiental; el $4 \%(n=2)$, a nutrición, y el $4 \%$ $(n=2)$, a medicina.

Según la orientación de las tesis se encontró que el $72 \%(n=34)$ correspondía a los aspectos preventivos, seguidos del correspondiente al de gestión, con el 17\% $(n=8)$. El $4 \%(n=2)$ de las tesis correspondieron a control de la enfermedad, y en menor proporción, a otras orientaciones (diagnóstico, brotes epidémicos e impacto económico).

Explorando propiamente la calidad de las tesis, el $90 \%(n=42)$ formularon la pregunta de investigación como pregunta per se o como objetivo, el $8 \%$ $(n=4)$ formularon el problema de modo implícito, y el $2 \%(n=1)$ no formuló la pregunta de investigación.

En cuanto a población y muestra, el $44 \%(n=21)$ de las tesis no precisaron la población, en tanto que en un $40 \%(n=19)$ la muestra no era representativa ni adecuada. El 13\% $(n=6)$ consignó la población como muestra, el $11 \%$ fue sólo adecuada, y el $6 \%$, sólo representativa.

En lo que concierne al análisis, un $72 \%(n=34)$ de las tesis lo realizaron de acuerdo con lo establecido en el apartado de materiales y métodos o comparando con otros estudios.

De las 47 tesis, sólo el 34\% $(n=16)$ analizaron y discutieron sus resultados según su plan de análisis, mientras que el 30\% $(n=14)$ se analizaron comparando los resultados con otros estudios, y el resto no analizaron sus resultados. Respecto a la conclusión, el 38\% $(n=18)$ respondieron al problema de investigación u objetivo del estudio, y sólo en 13 tesis (27\%) las conclusiones tuvieron un análisis previo.

Según la escala de calificación, el $66 \%(n=31)$ del total de las tesis correspondieron al calificativo de no aceptable, en tanto que el $11 \%(n=5)$ fueron aceptables y el $23 \%(n=11)$ se consideraron excelentes. De éstas, cuatro correspondieron a la maestría en epidemiología, y otras cuatro, a la maestría 
Figura. Distribución de la calidad de las tesis según la denominación de la maestría.

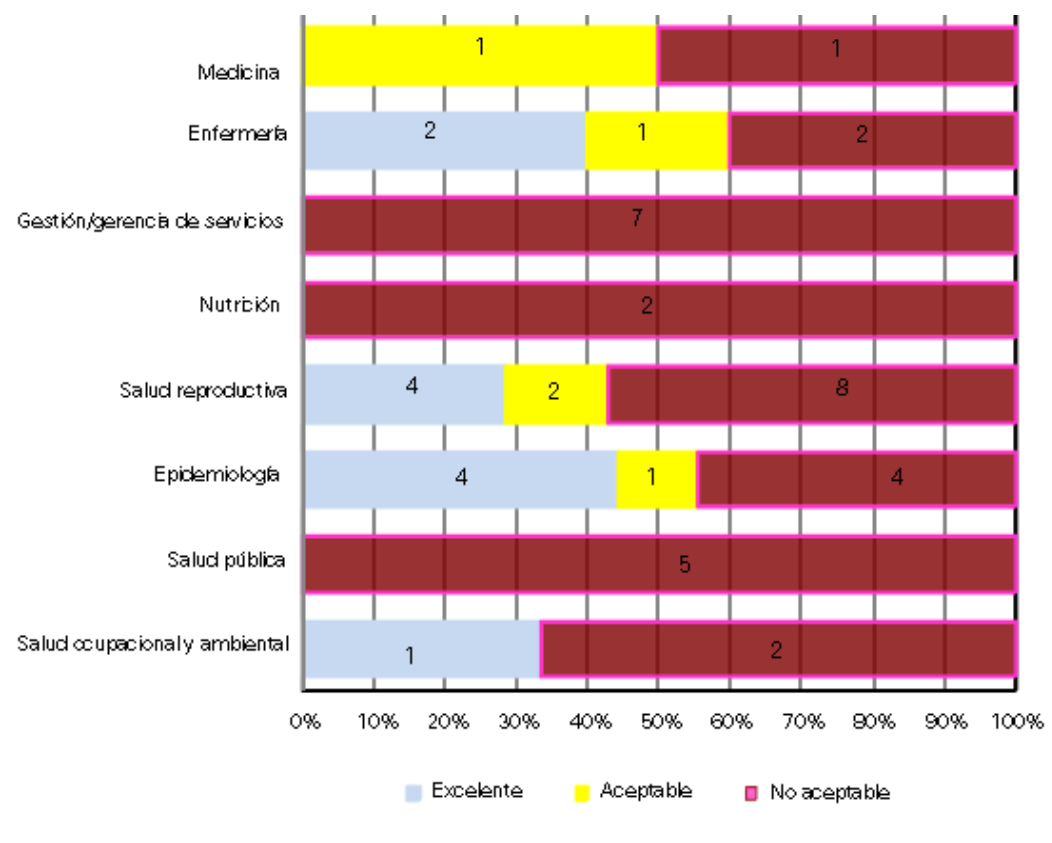

en salud reproductiva. De las 31 tesis no aceptables, el 23\% correspondieron a la maestría en gerencia de servicios de salud (Figura).

Finalmente, en cuanto a la relevancia social, 32 tesis (68\%) fueron relevantes; de ellas, la mitad fueron útiles para más de 500 personas, y las otras 16 , para el grupo comprendido entre 50 y 500 personas; sólo 10 tesis (21\%) fueron irrelevantes. En cinco tesis no se pudo determinar la relevancia por ausencia de información.

\section{Discusión}

El grado de magister es el grado académico intermedio que otorga la universidad. En Perú, y en la mayoría de países, dicho grado es una condición necesaria para hacer carrera universitaria. Las maestrías están concebidas para obtener una formación del bachiller que le permita con posterioridad llevar a cabo investigaciones de calidad. Sin embargo, muchos siguen la maestría con la intención de desarrollar su profesión al más alto nivel de competencia para lograr o mantenerse en un determinado campo laboral. Mendoza [1] clarifica esta situación en su ensayo sobre los grados académicos. Este razonamiento puede ser la explicación a los re- sultados de la presente investigación; primero discutiremos sus indicadores y, con posterioridad, la calidad propiamente dicha.

En las tesis analizadas, el problema fue el único indicador que la mayoría de los maestrandos presentó apropiadamente, lo que implica que el problema científico sí se formuló de forma correcta. Como se sabe, el problema de investigación es el inicio del proceso de investigación científica. Nuestro hallazgo de un $90 \%$ con un problema de investigación bien formulado en las tesis es similar al encontrado por Sanabria y Bullón [6] en su estudio de evaluación de las tesis de enfermería en la Facultad de Medicina de la UNMSM; en la evaluación de las tesis de bachiller en medicina de la UNSAAC se encontró que sólo el 3,2\% no definían el problema científico [8].

En el caso de la población y la muestra, no deja de ser llamativo que menos de la mitad de las tesis evaluadas en nuestro estudio no describan la población y, por tanto, no denoten la representatividad de la muestra; igualmente es llamativo que las muestras no fueran adecuadas en una proporción similar, es decir, el tamaño de la muestra correspondiente no se calculó de manera apropiada. En el estudio de las tesis de enfermería de la UNMSM [6], la muestra se describió apropiadamente en el 50\% de ellas. La evaluación de estudiantes de medicina en la UNSAAC demostró que el $20 \%$ de las 125 tesis estudiadas no comunicaron el tamaño de la muestra [8], porcentaje menor que nuestro hallazgo. A pesar de que los estudios cualitativos son cada vez más utilizados en salud pública $[9,10]$, ninguna tesis se realizó con la metodología cualitativa de investigación y, por tanto, no hubo muestras obtenidas por saturación teórica, propias de los estudios de investigación cualitativa.

En lo que concierne al análisis, nuestros hallazgos son mejores que los encontrados en la evaluación de las tesis de medicina $[5,8]$ y de enfermería [6,7]. Sin embargo, un indicador de análisis correcto menor del $80 \%$ no parece ser el mejor resultado. El análisis correcto implica que éste se haya hecho según el plan de análisis señalado en el apartado de materiales y métodos fundamentalmente, o bien, que se hayan establecido comparaciones con los resultados de otros investigadores en la discusión. Estos resultados invitan a mejorar los cursos de metodología de la investigación científica y estadística aplicada a la investigación que se dictan en las maestrías relacionadas con la salud pública.

Más comprometedor es lo concerniente a la conclusión, pues el porcentaje de presentación correcta es similar a la cifra encontrada, por debajo del $50 \%$, 
en tesis de enfermería en sendas universidades [6,7]. La tesis de Salazar [11] sobre la calidad de las investigaciones realizadas para obtener el título de médico especialista en la UNMSM demostró que el $83 \%$ era congruente con los objetivos y que algunas no presentaban conclusiones. Se aprecia entonces que tanto las investigaciones multidisciplinarias de posgrado conducentes al grado de magister como las realizadas exclusivamente por médicos tienen carencias en el indicador conclusión. Como se sabe, el nuevo conocimiento al que se llega tras haberse aplicado el método científico se describe en la conclusión. Por tanto, a nuestro juicio, se puede considerar que es el indicador más importante. Una mala conclusión refleja una tesis de baja calidad que, aunado a una pobre cultura de graduación, lleva a que la producción de nuevo conocimiento por tesis de maestría no aporte nada sustancialmente al desarrollo nacional. $\mathrm{Al}$ respecto, las tesis de maestría sustentadas en la UPG de la Facultad de Medicina no guardan una proporción con el número de maestrandos, lo cual es fácil de demostrar por las tesis publicadas (cibertesis) en la biblioteca virtual de la universidad correspondiente. Esta baja proporción de tesis en relación a los aspirantes a maestrías relacionadas con la salud pública se observaba también en universidades argentinas hasta el año 2002 [12].

En relación a la evaluación propiamente de la 'calidad' de las tesis, nuestros hallazgos de tesis excelentes son mejores que lo comunicado por investigadores en la UNSAAC $[5,8]$, quienes encontraron un $13-19 \%$ de excelencia en las tesis presentadas entre 1989 y 1993 en estudiantes de medicina, muy similar también a lo comunicado por Sanabria [6] en la Escuela de Enfermería de la Facultad de Medicina. Un mayor porcentaje $(19,4 \%)$ de excelencia de las tesis de medicina fue comunicado en 1996 por Nuñez-Sánchez y Kala-Conza [5]. En nuestro estudio es meritorio que, de las 11 tesis excelentes, la mayoría correspondieran a las maestrías en epidemiología y salud reproductiva. Los hallazgos bien pueden reflejar el nivel de conocimientos de epistemología y metodología de la investigación conforme a lo mencionado por Piscoya [13] y Kredert [7], lo que denota la necesidad de reforzar dichas áreas del saber. Resulta llamativo que de las 31 tesis no aceptables, aproximadamente la cuarta parte correspondan a la maestría en gerencia de servicios, lo que debe servir a los responsables de la conducción de la maestría para propiciar una autoevaluación al respecto.

Los hallazgos en relación a la calidad de las tesis en esta investigación inducen a lanzar una hipótesis: la calidad de las tesis de maestría en la UPG se correlacionan con la calidad de la docencia universitaria en investigación. Un estudio realizado en los años ochenta entre los médicos docentes de la Facultad de Medicina de la Universidad Nacional de Trujillo demostró insuficiencias en la parte metodológica de sus investigaciones [14]; se demostró que los indicadores mejoraron tras asistir a un curso de metodología de investigación científica. El problema es también estudiantil, pues se han encontrado 'vacíos' en cuanto a la actitud de los estudiantes hacia los cursos de investigación que se dictan en la Escuela de Enfermería de la Facultad de Medicina de San Fernando [15]. Esta situación podría explicar un error ab initio para el logro de la excelencia en las tesis estudiadas. Una investigación realizada en la Facultad de Educación de la UNMSM por Barrientos [16] demostró que sus aspirantes a la maestría no se encontraban capacitados para presentar un proyecto de investigación y que los docentes de la maestría correspondiente tenían un perfil académico medio. Otra investigación realizada en la Universidad Nacional de Trujillo con un grupo de 54 médicos estudiantes de posgrado en segunda especialización, por razones obvias, recomendó la necesidad de incluir cursos de epistemología en el currículo médico de pre y posgrado [17]. En otras palabras, no sólo los estudiantes son los responsables de la calidad de sus tesis, sino en alguna medida también los docentes universitarios.

La investigación es el motor que hace avanzar el conocimiento en todos los campos del desarrollo que interesan al ser humano para su vida, y uno muy especial es el de la salud. La formación de investigadores y de equipos de investigación es la herramienta imprescindible para el avance. En este proceso, el binomio docente-dicente tiene un peso específico. De superarse los problemas epistemológicos en el nivel docente y estudiantil, las tesis de investigación en el pre y posgrado contribuirán a incrementar el acervo científico de los estudios relacionados con la salud pública y la salud en general en Perú. Recordemos que dichos estudios están muy venidos a menos, según Chabés-Suárez et al [18], y que presentan una baja proporción coste-efectividad de la inversión, según Murillo y Rodríguez-Tafur [19].

En cuanto a la relevancia social, huelgan comparaciones toda vez que no se han encontrado estudios previos que tengan en cuenta dicho indicador, acuñado por nosotros; se espera que la comunidad científica refuerce o rechace esta propuesta. No hay una diferencia evidente en lo que respecta a trabajos relevantes desde tiempo atrás, pero debería preocupar su tendencia negativa. Muchas veces se toma la relevancia de modo general, refiriéndose a 
la importancia del estudio, que no es el caso en nuestra investigación. El estudio realizado en la UNSAAC [5] encontró un 77\% de investigaciones de importancia, aunque con el sentido de utilidad general y no propiamente en cuanto a su relevancia social, como se refiere este estudio.

Estos resultados permitirán a los responsables de la UPG, y particularmente a los coordinadores de las maestrías aludidas, precisar errores, anticipar tendencias y reorientar políticas educativas según lo establecido en su momento por Nogueira [20] sobre la evaluación de los recursos humanos en salud. Los resultados deber ser útiles a las autoridades correspondientes para mejorar la calidad de las futuras tesis de maestría de dicha unidad, situación que debe comprometer también a los docentes. Sólo así se fortalecerá el nivel académico del posgrado de la Facultad de Medicina de la institución superior que identifica a Perú en el mundo.

En conclusión, las tesis de maestría relativas a salud pública de la UPG de la Facultad de Medicina evaluada son, en su mayoría, socialmente relevantes, y en su minoría, excelentes o aceptables en cuanto a calidad metodológica. Se sugiere la necesidad de crear un programa de reforzamiento orientado a mejorar el proceso de formación y práctica de la investigación científica en el nivel de maestría en las áreas de salud pública de la UPG, particularmente de la maestría en servicios de salud.

Bibliografía

1. Mendoza P. Los grados académicos. Surgimiento y evolución. Anales de la Facultad de Medicina 2000; 61: 278-84.

2. Narváez R. Evaluación y medición de resultados. Revista Universitaria de la Universidad Nacional de Trujillo. Edición Extraordinaria Homenaje a Trujillo y Sesquicentenario de la Independencia Nacional. Trujillo, Perú; 1971.

3. Falcón P, Olaya G, Alpaca H. Evaluación de las tesis de grado de la Facultad de Medicina de Trujillo. Trujillo: Dirección Universitaria de Investigación, Universidad Nacional de Trujillo; 1978.

4. Veneros J, Risco R, Chávez A. Evaluación de las tesis de grado de bachiller en Ciencias Biológicas. REBIOL 1983; 3: 239-48.

5. Nuñez-Sánchez G, Kala-Conza E. Evaluación de la calidad de las tesis de pregrado desarrolladas en la Facultad de Medicina Humana de la UNSAAC, en Universidad San Antonio Abad del Cuzco, 1996 [tesis de Bachiller en Medicina]. Cuzco, Perú: Universidad San Antonio Abad del Cuzco; 1997.

6. Sanabria H, Bullón L. Exploración de la calidad de las tesis de enfermería de la Facultad de Medicina de San Fernando. Universidades 2000; 20: 27-34.

7. Kredert S. Correlación entre enseñanza de la investigación y rigor científico de las tesis de bachiller en la Facultad de Enfermería de la Universidad Nacional del Centro [tesis de Maestría en Enfermería]. Lima: Universidad Nacional Mayor de San Marcos; 1987.

8. Saco S, Quispe J. Diagnóstico de las tesis para optar por el título profesional de Médico Cirujano entre 1989 y 1993 en la Facultad de Medicina de la UNSAAC. Revista de la Facultad de Medicina de la UNSAAC 1992; 3: 19.

9. Sanabria H. Reflexiones para la investigación cualitativa en salud pública (I). Rev Peru Enf Inf Trop 2001; 1: 54-7.

10. Sanabria H. Reflexiones para la investigación cualitativa en salud pública (II). Rev Peru Enf Inf Trop 2001; 1: 106-12.

11. Salazar MA. Calidad de los trabajos de investigación que se realizan para optar el título de especialista en medicina humana de la UNMSM en el periodo 2001-2004 [tesis de Maestría en Docencia e Investigación en Salud]. Lima: Facultad de Medicina de la Universidad Nacional Mayor de San Marcos; 2007.

12. Romano M, Tobar F. La investigación dentro de las maestrías en salud pública en Argentina. In Borrel R, Rovere M. La formación en el postgrado en salud pública, Nuevos desafíos, nuevos caminos. 1 ed. Buenos Aires: OPS/OMS; 2004.

13. Piscoya L. Qué es epistemología? Alma Máter 1994; 6: 13-22.

14. Falcón P, Sanabria H, Zavaleta V. Eficiencia del Taller de Metodología de la Investigación Científica. Libro de Resúmenes de las Primeras Jornadas de Investigación y Docencia de la UNT. Trujillo, Perú, 14-15 de septiembre de 1981.

15. Miguel YM, Peñaranda RM. Actitudes de los estudiantes de enfermería de la UNMSM hacia el curso de Investigación en Enfermería y su relación con los factores personales e institucionales [tesis de Bachiller en Enfermería]. Lima: Universidad Nacional Mayor de San Marcos; 1994.

16. Barrientos E. El nivel de formación académica de los maestristas de la Unidad de Posgrado de Educación y su relación con el perfil, currículo y sus docentes. Revista de Investigación Educacional 1998; 1: 56-68.

17. Falcón MP. La situación de la epistemología en nuestro medio. Conceptos de docentes y residentes de medicina sobre algunos principios de la teoría de la ciencia. Revista Médica de Trujillo 1997; 2: 7-13.

18. Chabés-Suárez A, Murillo JP, Huicho L. Diagnóstico de la investigación biomédica en el Perú. Comunicación preliminar. Anales de la Facultad de Medicina 1997; 58: 199-200.

19. Murillo J, Rodríguez-Tafur J. Evaluación del costo-efectividad de la inversión estatal en investigación en ciencias de la salud. Alma Máter 1998; 16: 121-30.

20. Nogueira R. Tendencias y perspectivas de la investigación sobre personal de la salud de las Américas. Educación Médica y Salud 1995; 19: 33-45. 